\title{
Alcances y desafíos de la Educación en Derechos Humanos en la formacion de terapeutas ocupacionales, a partir de la percepcion de los estudiantes
}

\author{
Alcance e desafios da Educação em Direitos Humanos na formaçâo dos
} terapeutas ocupacionais, da percep̧̧ão dos estudantes

Scope and challenges of Education in Human Rights in the formation of occupational therapists, from the perception of students

Cristian Mauricio Valderrama Núñez ${ }^{\mathrm{a}}$ (D)

${ }^{a}$ Facultad de Ciencias de la Rehabilitación, Universidad Nacional Andrés Bello, Concepción, Chile.

Cómo citar: Valderrama Núñez, C. M. (2020). Alcances y desafíos de la Educación en Derechos Humanos en la formacion de terapeutas ocupacionales, a partir de la percepcion de los estudiantes. Cadernos Brasileiros de Terapia Ocupacional. 28(1), 26-49. https://doi.org/10.4322/2526-

8910.ctoAO1828

\begin{abstract}
$\underline{\text { Resumen }}$
El objetivo de este texto es describir el alcance y los desafíos de la Educación en Derechos Humanos en la carrera de Terapia Ocupacional de la Universidad Andrés Bello en Chile, a partir de la percepción de los estudiantes del primer ańo de la carrera de 2018. Para ello, se desarrolla una investigación con un enfoque fenomenológico descriptivo, se utiliza un cuestionario para producir la información, que es analizada por medio de estadística descriptiva y análisis de contenido semántico. Participan 277 estudiantes de las ciudades de Viña del Mar, Santiago y Concepción, que participaron y aprobaron durante el primer semestre de 2018 dos asignaturas que incluyen contenidos de Derechos Humanos en sus programas de estudios. Los principales resultados indican que la Educación en Derechos Humanos permite al estudiante desarrollar una perspectiva más amplia y compleja, presentando una visión crítica de sí mismo y de la realidad social. Al mismo tiempo, les proporciona una comprensión de su futura práctica profesional, como una posibilidad de crear una comunidad y una sociedad más justa, digna y solidaria. Los desafíos son incorporar contenidos y metodologías a la formación profesional que permitan reaprender las condiciones del capitalismo neoliberal que produce injusticias sociales, buscando alternativas para su superacion, entre ellas feminismo, descolonialidad y soberanía comunitaria. Por ultimo, parece necesario repensar los derechos humanos como fundamento
\end{abstract}


de otras éticas posibles, para la construcción de una ciudadanía democrática, desde una perspectiva intercultural.

Palabras-clave: Educación, Capacitación Profesional, Derechos Humanos, Terapia Ocupacional.

\section{$\underline{\text { Resumo }}$}

O objetivo deste texto é descrever o escopo e os desafios da Educação em Direitos Humanos na carreira de Terapia Ocupacional da Universidade Andrés Bello, no Chile, com base na percepção dos alunos do primeiro ano do curso, em 2018. Para isso, desenvolveu-se uma pesquisa mista, com abordagem fenomenológica descritiva, por meio de um questionário para produzir a informação, analisado através de estatística descritiva e análise de conteúdo semântico. Participam 277 estudantes das cidades de Viña del Mar, Santiago e Concepción, que foram estudantes aprovados, durante o primeiro semestre de 2018, em dois temas que incluem conteúdos de Direitos Humanos em seus programas de estudos. Os principais resultados indicam que a Educação em Direitos Humanos permite ao aluno desenvolver uma perspectiva mais ampla e complexa, apresentando uma visão crítica de si e da realidade social. Ao mesmo tempo, proporcionou-lhes uma compreensão da sua futura prática profissional, como uma a possibilidade de criar uma comunidade e uma sociedade mais justa, digna e solidária. Os desafios são incorporar conteúdos e metodologias à formação profissional que permitam reaprender às condiçóes do capitalismo neoliberal que produze injustiças sociais, buscando alternativas para sua melhoria, entre elas, feminismo, descolonialidade e soberania comunitária. Simultaneamente, repensar os direitos humanos como fundamento de outras possíveis éticas, na sua compreensão, a partir de uma perspectiva intercultural para a construção de uma cidadania democrática.

Palavras-chave: Educação, Formação Profissional, Direitos Humanos, Terapia Ocupacional.

\section{$\underline{\text { Abstract }}$}

The purpose of this text is to describe the scope and challenges of Human Rights Education in the career of Occupational Therapy at Universidad Andrés Bello in Chile, based on the perception of first-year students of the 2018 race. For this, a mixed research is developed, with a descriptive phenomenological approach, a questionnaire is used to produce the information, which is analyzed through descriptive statistics and semantic content analysis. Participants 277 students from the cities of Viña del Mar, Santiago and Concepción, which studied and approved, during the first semester of 2018, two subjects that include content of Human Rights in their study programs. The main results indicate that Human Rights Education allows students to develop a broader and more complex perspective, displaying a critical view of themselves and of the social reality; At the same time, it provides them with an understanding of their future professional practice, as a possibility to create a more just, dignified and supportive community and society. The challenges are to incorporate contents and methodologies to professional training that allow relearning the conditions of neoliberal capitalism that produces social injustices, seeking alternatives for their improvement, among them feminism, decoloniality and community sovereignty. And at the same time, to rethink human rights as the foundation of other 
possible ethics, in the understanding of these, from an intercultural perspective for the construction of a democratic citizenship.

Keywords: Education, Professional Training, Human Rights, Occupational Therapy.

\section{Introducción}

Este documento da cuenta de los resultados de una investigación sobre la formación inicial en materias de Derechos Humanos (DDHH), de los estudiantes de la carrera de Terapia Ocupacional $(\mathrm{TO})^{1}$ de la Universidad Andrés Bello (UNAB); la cual el año 2018 implementa una innovación curricular, cuya intención es instalar el tema de los derechos humanos en el corazón del currículo (Magendzo, 1998).

En Latinoamérica, la mayoría de las mallas curriculares de TO están dominadas por las asignaturas de carácter biológico, y las asignaturas de ciencias humanas y sociales están presentes en pequeña cantidad (Bianchi \& Malfitano, 2017).

Para este estudio es importante dar a conocer que, a diferencia de lo recién planteado, uno de los aspectos centrales de la innovación curricular de TO en la UNAB, es que el plan de estudio contiene una cantidad menor de asignaturas vinculadas a las ciencias biológicas, y un mayor número de asignaturas relacionadas a las ciencias sociales y humanas. Esto es relevante, porque los contenidos de DDHH se incorporan en asignaturas que pertenecen a estas últimas ciencias, así como a las asignaturas disciplinares (Universidad Andrés Bello, 2017).

Dicho lo anterior, este estudio tiene como objetivo describir los alcances y desafíos de la Educación en Derechos Humanos (EDH) en la carrera de TO, a partir de la percepción de los estudiantes de primer año, que recibieron formación en DDHH el primer semestre del año 2018. Los estudiantes cursaron 2 asignaturas que incorporan los $\mathrm{DDHH}$ en sus contenidos, estas son: "derechos humanos" y "rehabilitación $e$ inclusión con un enfoque de derechos humanos". Este estudio presenta los resultados orientados a la experiencia de la primera asignatura recién mencionada.

Esta asignatura se incluye, no solo por los contenidos que aborda, sino que, además implementa algunas de las metodologías que proponen Vega \& Nogueira (2018) para la EDH en la educación superior; entre ellas, análisis de casos, debates, foros, trabajos de investigación, sesiones expositivas, explicativas y demostrativas de contenido.

El documento comienza describiendo la relevancia de la EDH y las iniciativas internacionales que fomentan su implementación. Posteriormente, describe la situación de la $\mathrm{EDH}$ en Chile, lugar en el cual se desarrolla la investigación. Se continúa exponiendo la situación de la EDH en la carrera de TO a nivel mundial y nacional.

\footnotetext{
${ }^{1}$ La TO en Chile es una carrera profesional que dura 5 años, se dicta en universidades públicas y privadas, así como en institutos profesionales. Este estudio se desarrolla en una universidad privada. Las matriculas el ańo 2009 eran en total 2.302, para el ańo 2018 corresponden a 10.968 matriculados, lo que significa una variación de 376.5\% en ese periodo de tiempo. Lo que da cuenta del creciente número de estudiantes de TO en Chile (Chile, 2018a).
} 
Finalmente, se describe cómo se desarrolló el estudio y sus principales resultados y conclusiones, enfocando principalmente a los desafíos en la formación de los terapeutas ocupacionales para el contexto latinoamericano actual.

\subsection{Educación en derechos humanos}

Los DDHH son aquellos derechos pertenecientes a todos los seres humanos por el solo hecho de ser humanos, son por tanto un imperativo ético/moral prioritario para hacer posible una vida digna (Navarrete Salas et al., 2015).

Los derechos sociales son todos aquellos derechos que persiguen mejorar las condiciones materiales de la vida y lograr una igualdad material entre todos los seres humanos (Estay, 2010). Los derechos sociales presentan distintas clasificaciones, pero en general se refieren a los derechos a la salud, la educación, el trabajo y la previsión social.

Cuando los derechos sociales se garantizan por parte del Estado y todos los ciudadanos pueden ejercerlos, podríamos señalar que se cristalizan los derechos humanos, dado que se materializan los principios éticos de dignidad e igualdad de las personas y comunidades.

Sin embargo, como plantean Murcia et al. (2007), el capitalismo neoliberal pone en riesgo el ejercicio de los derechos sociales y los $\mathrm{DDHH}$, por las siguientes razones: exacerba la regulación de la vida social por parte del mercado, prioriza la libertad individual por sobre la libertad colectiva, fomenta el derecho a la propiedad privada por sobre cualquier otro derecho, opera por criterios económicos y de productividad en temas eminentemente humanos, es decir, pone precio a aspectos que no pueden tener precio; por último, disminuye el rol proteccionista y democrático del Estado y de la ciudadanía.

El efecto es que si el Estado pierde la capacidad de garantizar los derechos sociales y si la ciudadanía deja de conquistarlos y ejercerlos, éstos se transforman en un bien de consumo, que solo pueden disfrutar quienes poseen los medios materiales para adquirirlos, aumentando las desigualdades e injusticias sociales. En este contexto de amenaza a las democracias actuales, por parte del capitalismo neoliberal, se torna fundamental la $\mathrm{EDH}$, como medio para la protección y fomento de los DDHH y la materialización de los derechos sociales.

La EDH busca concientizar sobre las responsabilidades individuales y colectivas en la promoción y protección de los $\mathrm{DDHH}$, pretende fomentar la reflexión y análisis críticos de las bases éticas de las democracias en el mundo, prevenir y ofrecer soluciones ante las vulneraciones y violaciones de los $\mathrm{DDHH}$, generar un cambio de percepciones y actitudes para ser coherentes con los principios valóricos de los DDHH. Al mismo tiempo, debe potenciar el ejercicio de la ciudadanía y la construcción de memoria histórica. La EDH es en esencia constructora de paz (Magendzo, 2003, 2008; Organización de Naciones Unidas, 2010; Sacavino, 2015; Vega \& Nogueira, 2018).

Para la Organización de Naciones Unidas (2011), la EDH es un proceso que debe estar presente durante todo el ciclo vital y concierne a todos los integrantes y sectores de la sociedad. Involucra todas las formas de educación posible formal o informal, tanto en el sector público como en el privado. 
En la Conferencia Mundial de DDHH de 1993, se determina que los Estados promoverán la inclusión de las temáticas de $\mathrm{DDHH}$, el derecho humanitario y la democracia en todos los planes de estudios de todas las instituciones de enseñanza académica y no académica (Organización de Naciones Unidas, 1993). Desde el año 2005 existe el programa mundial de $\mathrm{EDH}$, que progresivamente incorpora en sus planes quinquenales a distintos grupos objetivos (Organización de Naciones Unidas, 2005, 2010, 2013).

En relación a la enseñanza superior, campo en el cual se desarrolla esta investigación, la Organización de Naciones Unidas (2010) considera centros de enseñanza superior a las Universidades u otros establecimientos de formación superior, acreditadas por las autoridades competentes del Estado. Este punto es relevante para los propósitos de este estudio, en tanto la UNAB institución en la cual se realiza el estudio, recientemente obtiene acreditación institucional por 5 años, otorgada por la Comisión Nacional de Acreditación (CNA) en Chile (Comisión Nacional de Acreditación, 2018). Por tanto, es una institución objeta de la responsabilidad de implementar y desarrollar la EDH.

\subsection{Educación en derechos humanos en Latinoamérica: el caso de Chile}

En Latinoamérica se comienza a hablar de EDH en la década de los años 80 del siglo XX, en un contexto de dictaduras militares; en ese marco, la EDH se expresa de manera no formal en sectores populares y promovidos por las organizaciones defensoras de los DDHH y de las Organizaciones No Gubernamentales (ONGs).

La EDH en América Latina está ligada al movimiento de la educación popular de Paulo Freire; por lo tanto, estaba pensada en generar conciencia y acción, para la transformación de las injusticias sociales y fomentar la solidaridad (Magendzo, 1998; Magendzo \& Toledo, 2015). Cuestión que, en la actualidad se torna imperiosa abordar dado que las injusticias sociales, pobreza y marginación de los pueblos latinoamericanos aún persisten.

Con el retorno de las democracias en América Latina, la EDH se fue incorporando paulatinamente a los currículos de la educación formal y se ha reforzado en la educación no formal (Magendzo \& Toledo, 2015). En países como Argentina, Brasil y Colombia, se impulsa la EDH en las nuevas constituciones post dictaduras, a través de los planes nacionales de EDH (Sacavino \& Candau, 2008; Restrepo, 2009; Fernández, 2016; Tavares, 2016). Tema aún pendiente en el caso de Chile.

Para este estudio, es fundamental situarse en la realidad chilena, ya que este país no cuenta con una legislación y política consistente para el fomento de la EDH. Luego del retorno de la democracia ha realizado algunos esfuerzos legislativos, uno de los más relevantes es la Ley General de Educación, que establece la obligación de promoción de los DDHH en los procesos educacionales (Azúa, 2011).

En el año 1990, la Comisión Nacional de Verdad y Reconciliación realiza un informe con sugerencias, entre las que se incluyen "[...] la incorporación de contenidos, habilidades y valores respetuosos de los derechos humanos en todos los niveles de la enseñanza formal e informal" (Instituto Nacional de Derechos Humanos, 2014, p. 3). Sin embargo, estas modificaciones han sido insuficientes, ya que Chile no 
cuenta con un Plan Nacional de EDH (Instituto Nacional de Derechos Humanos, 2012).

En el plan nacional de DDHH, se contemplan algunos avances en EDH, entre ellos, la elaboración de un protocolo que permita la creación de una política pública de educación con enfoque de DDHH, y la incorporación de este enfoque en los planes de estudios de las carreras de la salud (Chile, 2018b). Esta investigación representa un esfuerzo por materializar la EDH en la carrera de Terapia Ocupacional, cuestión que sintoniza con la propuesta recién mencionada.

Según el Instituto Nacional de Derechos Humanos (2012), los desafíos de la EDH en la educación superior, estarían relacionados a la falta de regulación del Estado sobre estas instituciones, al alto grado de autonomía de ellas y a la no incorporación de la EDH en aquellas estrategias de regulación y/o desarrollo de las Universidades.

Nash (2011) señala que de un total de 20 Universidades públicas y privadas, el $55 \%$ ofrece alguna asignatura específica sobre $\mathrm{DDHH}$, siendo la mitad de ellas obligatorias, escasamente se vinculan a temas de grupos vulnerados y de preferencia se vinculan al campo jurídico.

En cuanto a los contenidos que abordan los programas de asignaturas de $\mathrm{DDHH}$, en carreras como derecho, periodismo y trabajo social. Silva (2014) señala que el $40 \%$ de los programas consideran los principios de la Igualdad y no discriminación. Los Programas de curso que consideran a los grupos vulnerados corresponden al $23 \%$, pobreza $3 \%$, discriminación $26 \%$, conflictos violentos $20 \%$ y situaciones históricas $40 \%$.

En relación a los dos estudios recién mencionados, esta investigación se desarrolla en un plan de estudio distinto, dado que la asignatura de derechos humanos es obligatoria y varios de sus contenidos están dirigidos a la vinculación de los DDHH con grupos vulnerados y a los principios de igualdad y no discriminación.

\subsection{Educación en derechos humanos en terapia ocupacional}

En el caso de la $\mathrm{EDH}$ en $\mathrm{TO}^{2}$, varios autores han señalado la relevancia de incorporar en la formación profesional y la investigación en TO el enfoque de derechos humanos (Mousavi et al., 2015; Hocking \& Townsend, 2015; Galheigo, 2011), y en lo posible que se encuentre presente de manera transversal en la formación disciplinar y profesional (Oyarzún et al., 2010). En este sentido, la innovación curricular de la carrera de Terapia Ocupacional de la UNAB, recoge plenamente estas propuestas, abordando los DDHH transversalmente en los 3 ámbitos que la componen: investigación, intervención y gestión (Universidad Andrés Bello, 2017).

A nivel de la educación superior, Jung et al. (2008) plantean que los programas de TO en sí mismos, cumplen un rol importante para fomentar el acceso a la inclusión

\footnotetext{
${ }^{2}$ Para establecer la relación de la EDH y TO, se asume que los DDHH están presentes de distinta forma en las actividades curriculares y extracurriculares de la formación de terapeutas ocupacionales en Chile, dado que varias Universidades la declaran en sus perfiles de egreso. Por ejemplo, en la Universidad de las Américas (2018) y en la Universidad San Sebastián (2018). Para efectos de este estudio, las investigaciones en docencias desarrolladas con estudiantes y las investigaciones realizadas por estudiantes de pregrado, se consideran como experiencias de EDH en TO.
} 
educativa, de aquellos que el sistema educativo históricamente ha discriminado. Hecho que se vincula al derecho social a educarse y ser educado.

En este mismo sentido, Whiteford (2017) señala la relevancia al acceso y participación en la educación superior de los grupos vulnerados, ya que, desde una perspectiva ocupacional sería una forma de inclusión social, que además permitiría la construcción de la identidad y mejoraría el estado socioeconómico.

A partir de los estudios recién mencionados se podría señalar que, al ejercer el derecho a la educación, se cristaliza lo que Fernández (2012) describe como ciudadanía, ya que las personas son reconocidas como titulares de derechos, que pueden acceder y ejercer derechos sociales garantizados por el Estado.

A la vez, al ejercer el derecho a la educación, a los sujetos se les reconoce el derecho humano a pertenecer y construir su identidad en una comunidad de pares, con los cuales hay un compromiso ético y moral recíproco, por participar activa y responsablemente en el desarrollo de una convivencia, que fortalezca democráticamente los aspectos políticos, sociales, culturales y económicos de esa comunidad (Fernández, 2012).

Por su parte, Piškur (2013) propone que en TO se debe considerar la participación de los sujetos de intervención y las organizaciones en la educación e investigación. Este tema es relevante en tanto, esta propuesta obligaría en la formación disciplinar, a incorporar desde una perspectiva de los DDHH el reconocimiento, respeto y dignidad de los sujetos de intervención, organizaciones y movimientos sociales, tanto en la docencia como en la generación del conocimiento.

Las investigaciones recién mencionadas, tratan temas vinculados a los DDHH como la inclusión social, la participación social y el reconocimiento de los sujetos de intervención. La diferencia con el estudio que se presenta, es que éste se refiere a la formación recibida por estudiantes de pregrado en contenidos propios de los DDHH.

En este sentido, Crawford et al. (2017) indican que los estudiantes de último año de $\mathrm{TO}$, al recibir $\mathrm{EDH}$ aumentan los conocimientos, habilidades y confianzas para trabajar no solo una práctica centrada en el cliente, sino que también las desigualdades sociales.

En Latinoamérica, Lopes et al. (2012) y Valderrama et al. (2015), plantean que la enseñanza en el campo social y situada en la realidad socio comunitaria, proporciona a los estudiantes de TO, la posibilidad de promover los principios de la democracia y el ejercicio de los DDHH. Este punto es crucial, ya que invita a comprender la formación en DDHH como una práctica social.

En Chile se podría señalar que la EDH en TO, se encuentra concentrada en los procesos de investigación de pregrado e incluye una variedad de temáticas de DDHH; entre ellas, la lucha por el derecho a la salud de una agrupación de padres y madres en Concepción (Alarcón et al., 2017).

El uso de lenguaje discriminatorio y no inclusivo en contextos escolares, hacia niños y nińas con discapacidad en la ciudad de Punta Arenas (Yupanqui et al., 2016). La exploración del impacto de una intervención de TO, desde la perspectiva de género y DDHH, en el vínculo entre mujeres privadas de libertad y sus hijos en la ciudad de Punta Arenas (Galindo et al., 2016).

Por su parte Dehays et al. (2013), estudian la vulneración de derechos a la cual están expuestas las mujeres con discapacidad, en el ejercicio de la maternidad, en la 
ciudad de Punta Arenas. A su vez Ortiz et al. (2014) estudian el impacto que genera el diagnóstico de VIH, específicamente los efectos negativos vinculados a los prejuicios y estereotipos, asociados al diagnóstico y sus efectos en el derecho al trabajo. Estos dos trabajos, han sido reconocidos como ganadores del concurso "cuenta tu tesis" 3

Por último, Espinoza \& La Pietra (2017) desarrollan en Chile un estudio con estudiantes de quinto ańo, quienes entregan una valoración positiva a la articulación entre aprendizajes en DDHH, generación de valores y actitudes y el desarrollo de aspectos éticos, a través de la metodología de enseñanza aprendizaje de servicio.

El año 2018 la Escuela de TO de la UNAB, implementa una innovación curricular, que busca formar terapeutas ocupacionales con una base valórica y ética sustentada en los DDHH, con un pensamiento crítico y que desarrollen procesos de intervención orientados a fomentar al ejercicio de la ciudadanía, la inclusión social, la participación y justicia ocupacional (Universidad Andrés Bello, 2017).

Este estudio se enfoca específicamente en el tema de los DDHH recién declarado. Para ello, se incluye a los estudiantes de primer ańo, cuestión que lo diferencia de los estudios anteriormente señalados, ya que todos ellos se enfocan en estudiantes en los últimos años de formación.

Dado que este estudio se realiza en 3 ciudades (Viña del Mar, Santiago y Concepción), en las que se dictó la asignatura de DDHH, para garantizar la homogeneidad en los contenidos y metodologías de enseñanza y aprendizaje, se confecciona y desarrolla un único programa de la asignatura para las 3 sedes. En este programa se abordan los siguientes contenidos:

- Unidad 1: Evolución histórica de los DDHH (Antecedentes históricos de los DDHH, Antecedentes previos a la declaración universal de los DDHH, Pacto internacional de derechos civiles, políticos, económicos, sociales y culturales, Concepción intercultural de los DDHH);

- Unidad 2: Principios transversales de igualdad y no discriminación (principio de igualdad, de no discriminación, Democracia y ciudadanía);

- Unidad 3: Violaciones de los DDHH y mecanismos de protección (Obligaciones del Estado, Criminología de las violaciones de los DDHH, Tipos de responsabilidad por violaciones de los DDHH. Sistemas nacionales, regionales y universales de protección de los $\mathrm{DDHH}$ );

- Unidad 4: DDHH en grupos vulnerables (derechos de la mujer, de los pueblos indígenas, de la diversidad sexual).

Para ejemplificar las metodologías de aprendizaje, se presenta lo que está diseñado en el programa de la asignatura, para la clase que aborda el de tema violaciones de los DDHH y mecanismos de protección. En el cual se desarrollan las siguientes actividades:

\footnotetext{
${ }^{3}$ El concurso Cuenta tu tesis en Derechos Humanos, lo realiza el INDH y tiene como principal propósito reconocer aquellos trabajos de titulación, desarrollados en el ámbito de los derechos humanos en Chile, de instituciones chilenas de educación superior acreditadas.
} 
a) Clase expositiva;

b) Revisión de un video: ejemplo de Violación de los Derecho humanos en México (Sinembargo TV, 2016);

c) Se entrega una guía de trabajo grupal, para analizar el contenido del video;

d) Se desarrolla un plenario para discutir la temática y recoger las ideas fuerzas.

La pregunta que se pretende resolver es: ¿Cuáles son los alcances y los desafíos de la EDH en la carrera de TO, a partir de la percepción de los estudiantes de primer año que recibieron formación en DDHH el primer semestre del año 2018? A continuación, se describirá de qué manera se desarrolló el estudio y las decisiones metodológicas que lo permitieron.

\section{Metodología}

Este trabajo se enmarca en una investigación mixta. El enfoque del estudio es fenomenológico descriptivo, ya que se propone describir las opiniones, percepciones y la forma de comprender el fenómeno de los estudiantes de TO (Gurdián-Fernández, 2007; Rivero, 2008; García \& García-Celay, 2015).

Para la producción de los datos, se confeccionó un cuestionario auto administrado, compuesto por tres partes: 1.- preguntas de identificación general (Cerón, 2006); 2.- encuesta basada en dos instrumentos estandarizados (Instituto Nacional de Derechos Humanos, 2011; Organización Mundial de Salud, 2015) y; 3.- tres preguntas abiertas. Este estudio se centra en la información producida por la encuesta y las preguntas abiertas.

El instrumento seleccionado se funda en la distancia entre los distintos participantes y el investigador, por la cantidad de estudiantes involucrados y por la facilidad de su aplicación.

Con respecto a la encuesta, son 26 afirmaciones que abordan 6 dimensiones relacionados a los DDHH: 1. percepción general de los DDHH; 2. igualdad y no discriminación; 3. situación de derechos humanos en Chile; 4. memoria y violación a los DDHH; 5. reconocimiento jurídico de las personas con discapacidad mental; 6. derecho a vivir en comunidad de las personas con discapacidad mental.

Para este estudio, se consideran los resultados obtenidos de las cuatro primeras dimensiones recién mencionadas, dado que contemplan los contenidos abordados en la asignatura de "derechos humanos". Los resultados de las dos últimas dimensiones se excluyen, dado que están relacionados a los contenidos abordados en la asignatura "rehabilitación e inclusión con un enfoque de derechos humanos", que escapan a los propósitos del presente escrito.

La encuesta utiliza una escala tipo Likert ordinal, atribuyendo valores numéricos a 4 puntajes positivos/negativos: muy de acuerdo, 4; de acuerdo, 3; en desacuerdo, 2; y muy en desacuerdo, 1.; ya que de esta forma permite ordenar las respuestas independientes de los atributos del estudiante que la contesta y desde donde la contesta, identificando la valoración positiva o negativa de la afirmación (Ospina Rave et al., 2005; Cerón, 2006). 
En relación a las preguntas abiertas, (1. ¿Cuál ha sido la importancia de recibir formación en temas de Derechos Humanos?; 2. ¿Cuáles son los temas de derechos humanos abordados en tu formación, que consideras más difíciles de comprender?; 3. ¿Cuál es el aporte de las temáticas de derechos humanos a tu futuro profesional?) Se realizaron para identificar y describir la opinión de los estudiantes, en torno a la importancia, dificultades y proyecciones en el ejercicio profesional, de la $\mathrm{EDH}$ en su formación profesional. De esa forma es posible, capturar los puntos de vista de los estudiantes (Gurdián-Fernández, 2007; Rivero, 2008). Desde las preguntas abiertas surgen 3 categorías emergentes: exigibilidad, construcción de ciudadanía, mecanismos de protección. Para este documento se incorporan las dos primeras en los resultados y análisis, ya que son las que presentan mayor relación con los propósitos de este estudio.

Los participantes fueron seleccionados de manera intencionada, dado que se establece como criterio de inclusión, que los estudiantes hayan cursado y aprobado durante el primer semestre del año 2018 la asignatura involucrada (Rivero, 2008). Este criterio se aplica, dado que daría cuenta que los estudiantes han adquirido los aprendizajes esperados de la asignatura seleccionada para el estudio.

La carrera de TO en la UNAB se dicta en 3 sedes: Santiago, Viña del Mar y Concepción. El estudio abarcó la totalidad de las sedes. Participaron de este estudio, un total de 277 estudiantes, ( $n=80$ de Viña del Mar, $n=126$ de Santiago, $n=71$ de Concepción), que representan el $75,27 \%$ de los potenciales participantes.

La aplicación del cuestionario se realizó presencialmente durante el mes de agosto, en el horario de clases del segundo semestre con el apoyo del docente a cargo de la asignatura respectiva. El instrumento se envió por correo electrónico, desde la sede de Concepción, donde reside el autor de este estudio, a las direcciones de carrera de Viña del Mar y Santiago para ser distribuida a los docentes. Luego de aplicado el cuestionario (en formato papel), cada sede envía los cuestionarios completados por servicio de mensajería.

Los cuestionarios se ordenan y agrupan por sede $(S)$, información incluida en el instrumento, asignándole un numero de acuerdo a la siguiente distribución: 1 para Viña de Mar, 2 para Santiago y 3 para Concepción; luego de estar ordenadas por sede, se les asigna un numero correlativamente del 1 al 277, para identificar las respuestas de cada participante $(\mathrm{P})$.

El análisis de las encuestas, se desarrolla a través de estadística descriptiva, se calcula la frecuencia y la media de las respuestas de cada categoría, que conforma cada una de las dimensiones exploradas. Los valores "muy de acuerdo" y "de acuerdo" se combinaron para determinar la percepción positiva general por categoría. Los valores "muy en desacuerdo" y "en desacuerdo", se combinaron para determinar la percepción negativa general por categoría.

Para determinar la percepción positiva o negativa de cada dimensión, se calcula la suma de las medias de las categorías que conforman dichas apreciaciones.

En relación a las preguntas abiertas, se realiza una matriz categorial para clasificar los contenidos de los textos por categorías, tanto para las categorías apriorípticas como para las categorías emergentes (García \& García-Celay, 2015). Se utiliza análisis del contenido semántico para esta clasificación, dado que interesa el sentido del texto, en 
tanto éste daría cuenta de las percepciones de los estudiantes sobre cada categoría de análisis (Amezcua \& Galvéz, 2002).

En relación a las consideraciones éticas, se adjuntó con el cuestionario, un consentimiento informado, el cual establecía la confidencialidad, voluntariedad y no maleficencia. Todos los participantes firmaron este documento.

Para garantizar el anonimato de los participantes, el cuestionario se responde de manera anónima y no recoge datos personales (nombre, teléfono, dirección personal o correo electrónico).

\section{Resultados}

En un primer momento se presentarán los resultados estadísticos descriptivos de las dimensiones abordadas en el cuestionario. Además, se utilizarán los discursos escritos de los estudiantes para ilustrar los datos estadísticos.

En un segundo momento se presentarán los resultados de las dos categorías emergentes de las preguntas abiertas, a través de los relatos de los estudiantes que las componen.

En cuanto a la dimensión percepción de los $\mathbf{D} \boldsymbol{D H} \boldsymbol{H}$, el 88,8\% de los participantes del estudio otorgan una valoración positiva a los $\mathrm{DDHH}$, lo cual es vinculado al hecho de haber recibido formación en ellos, como lo señalan los siguientes relatos:

Poder respetar y hacer valorar mis derechos y el de los demás, es muy importante educarse (S1. P9).

Hoy en día se nos hace costumbre que nos vulneren nuestros derechos, y esta implementación nos ayuda a empoderarnos (S1. P88).

Comprendiendo los DDHH es que podemos habitar la Tierra de forma pacifica y con una sana convivencia (S3. P243).

Que se implemente en el primer año de la carrera, nos dan las bases de cómo actuar, actuar siempre con una perspectiva de DDHH, ya que somos agentes de cambio (S2. P88).

En esta dimensión, la categoría que en las encuestas alcanza el promedio más bajo de percepción positiva, es la que señala "me parece que es preferible una sociedad donde se respeten todos los derechos, aunque signifique que pueda existir algún desorden social y económico", con un $69,9 \%$. Esto podría estar reflejando, la idea que una sociedad donde se respetan los DDHH, es más propensa a la ingobernabilidad y a la inestabilidad económica. Por ende, se plantearía una incompatibilidad entre los DDHH y el desarrollo social y progreso económico.

Estos resultados refuerzan la idea de Larraín (2011), quien describe que el actual capitalismo neoliberal imperante, desconfía de la democracia y de los DDHH, dado que estos serían una amenaza para el orden espontáneo del mercado. El resultado recién señalado, podría estar dando cuenta de la influencia cognitiva y simbólica del neoliberalismo en los estudiantes. Por tanto en TO, es necesario ofrecer una discusión 
al respecto para comenzar a revertir esta situación en la formación profesional, cuestión que se aborda más adelante en este texto.

Simultáneamente en las respuestas a las preguntas abiertas, las dificultades que se presentan para comprender los DDHH, se relacionan a la confusión que genera el hecho de que existan obstáculos para ejercer los derechos, así como el hecho que existan prácticas que los vulneran, más aún cuando los $\mathrm{DDHH}$ son percibidos y significados como fundamentales para la sociedad, como lo señalan las siguientes frases:

[...] falta educación sobre los $\mathrm{DDHH},[\ldots]$ que no se respetan, a cada instante los estamos vulnerando (S2. P198).

[...] sigue habiendo prácticas que atentan contra estos derechos [educación y salud], privatizando todo, sin una fiscalización adecuadas ni de la ciudadania ni del Estado (S2. P144).

Comprender por qué los DDHH aún no son respetados sabiendo que son de suma importancia, que nos hace tener un mundo en paz, todos los que vinculan a los DDHH con la politica y la religión (S3. P202).

En la dimensión igualdad $\boldsymbol{y}$ no discriminación, los estudiantes presentan un promedio de $76,5 \%$ de apreciación positiva, en las categorías ahí abordadas.

La categoría con el promedio más alto de percepción negativa, corresponde a la vinculada a las mujeres, en la cual un $38.6 \%$ de los estudiantes, considera que la mujer puede trabajar, pero sin descuidar a los hijos.

Estos resultados, son similares con lo investigado por Sarmiento et al. (2018), quienes concluyen que los estudiantes presentan arquetipos y estereotipos automatizados, como asociar el sexo de la mujer con el rol social de madre. Es decir, en los estudiantes de TO está presente, el arquetipo de que las mujeres deben ser madres antes que trabajadoras.

Dicho lo anterior, es posible advertir que en la formación de TO, los temas vinculados al género femenino y los feminismos estarían pendientes de profundizar. Cuestión que se considera relevante ahondar más adelante en la discusión de este documento.

En esta misma dimensión, en los relatos obtenidos a partir de las preguntas abiertas, predomina la percepción positiva que los $\mathrm{DDHH}$, son necesarios para una convivencia más humana, el respeto a la diversidad y a la construcción de un país más justo:

Nos ayudan a nivel pais y personal tener un trato igualitario con las personas (S1. P6).

Debemos respetar los derechos de cada persona, sin importar su condición socioeconómica (S2. P104).

Encuentro que es sumamente importante para comprender, tolerar, respetar a las demás personas de manera igualitaria (S3. P221). 
Podemos crear conciencia de todos los hechos de vulneración y fomentar el respeto que es algo importante en una convivencia humana, además los DDHH ayudan a la comunidad, a las personas, al pais a crear justicia y condiciones de igualdad (S1. P67).

Desde lo que señalan Córdova \& Galheigo (2015), la EDH en Terapia Ocupacional aportaría en la construcción de una ciudadanía legitimada en los principios que los DDHH promueven (dignidad, respeto, solidaridad, entre otros), en una ciudadanía legitimada en la propia comunidad y en su propia capacidad deliberativa.

Por otra parte, en esta dimensión lo más complejo de comprender para los estudiantes, está vinculado al hecho de la inalienabilidad de los DDHH, es decir, entender que una de las cualidades de los $\mathrm{DDHH}$, es que legalmente no se les pueden negar a ninguna persona. Sobre todo, pensando en aquellos sujetos que han cometidos delitos o crímenes de lesa humanidad, como lo señalan los siguientes textos:

El hecho de empatizar o proteger los $\mathrm{DDHH}$ de alguien que ha vulnerado los de alguien más (S1. P18).

El tema de aquellas personas que hacen cosas malas [cárcel], aun asi, el Estado y gobierno los defienden y los cuidan (S2. P62).

Encuentro que esto no debiese pasar, ya que ellos mismos violan los DDHH (S2. P92).

El tema de las personas privadas de libertad, que a pesar de haber cometido delito aun asi son personas y sujetos de derechos (S1. P54).

Con respecto, a los beneficios que otorgaría la $\mathrm{EDH}$ en la práctica profesional, los estudiantes señalan que les proporciona la capacidad para respetar los derechos de los sujetos de intervención, para un trato digno, respetuoso e inclusivo, y por último, para vincularse con el otro, reconociendo y reconociéndose a sí mismo con ese otro:

Es fundamental, ya que tratamos con gente de diferentes etnias, edades, sexo, etc, y todos por igual deben tener un trato digno (S1. P29).

Hacer valer los derechos que tenemos todos por el hecho de ser persona, sin importar la situación en la que esté [migración, PsD, escasos recursos], para que pueda gozar de buen vivir (S2. P82).

[...] con un trato igualitario por el hecho de ser personas, pero a la vez con un trato diferenciado porque somos seres humanos con distintos contextos de vida, cultura, sexualidad, entre otros (S3. P202).

La EDH promueve la posibilidad de que los estudiantes reconozcan la importancia de construir una sociedad basada en la dimensión relacional de los DDHH, es decir, basada en el reconocimiento del otro, en el respeto de la diversidad, entre otros (Córdova \& Galheigo, 2015). 
En cuanto a la dimensión situación de los $\mathbf{D D H H}$ en Chile, los resultados dan cuenta de una consideración negativa por parte de los estudiantes, quienes en un $87,6 \%$ consideran que en Chile la situación de los DDHH es desfavorable. La categoría menos valorada con un $94,9 \%$ de preferencias, es que en Chile no se respetan los $\mathrm{DDHH}$ de todas las personas. La segunda categoría menos valorada con un $92,4 \%$, es que en Chile la ley no se aplica a todos por igual.

Las opiniones de los estudiantes, refuerzan las cifras recién señaladas en cuanto a la situación desfavorable en Chile de los $\mathrm{DDHH}$, como lo señalan los siguientes relatos:

Tener el conocimiento necesario sobre el tema, ya que lamentablemente, en nuestro pais pareciera ser algo poco relevante (S1. P34).

El derecho a la vivienda y la salud en Chile, en Chile por la situación económica, algunos no pueden ejercer estos derechos (S2. P91).

Conocer la realidad nacional y notar la ignorancia que hay con respecto a los DDHH y sus instituciones (S3. P225).

Los estudiantes presentan una percepción crítica en torno a la memoria y violación de los $\mathbf{D D H H}$, ya que las percepciones sobre los criterios que la componen se distribuyen de la siguiente manera: el 94,2\% considera que en dictadura se violaron brutalmente los DDHH de las personas, el 85,6\% considera que estas violaciones es un tema que no hay que olvidar, el $84,5 \%$ considera que ha existido demasiada impunidad con la mayoría de los violadores a los DDHH, y por último, el 68.2\% cree que el país no ha aprendido lo suficiente de lo ocurrido. Por ende, este sería un tema pendiente como país, que requiere una política nacional al respecto.

Esta capacidad crítica, los estudiantes la atribuyen a que durante el proceso de EDH recibido, toman conocimiento y son conscientes de la historia acontecida en dictadura:

Muchisima, ya que en el colegio no tenía ideas de que muchos DDHH fueron violados y todo lo que paso, [...] pude saber los hechos que pasaron en el pais y en otros lugares (S1. P45).

Crear conciencia a partir de hechos de injusticia, además de conocer nuestro pasado, para saber quiénes somos hoy (S1. P17).

Soy de una generación en la que se considera bueno no remover el pasado, y creo que es muy necesario hacer todo lo contrario (S3. P235).

Nos hizo, de alguna forma, "abrir los ojos" y conocer sobre el pasado y las violaciones a los DDHH ocurridos en distintos lugares (S3. P265).

Al mismo tiempo, el hecho que los estudiantes tomen conocimiento de los mecanismos de violación de los DDHH durante la dictadura militar, produce una sensación de impunidad e injusticia debido a la falta de verdad y justicia que al día de hoy existe, como lo señalan los siguientes relatos: 
La impunidad de casos en contra de los DDHH, icómo es posible que sigan permitiendo estos tipos de violaciones a los DDHH? (S1. P39).

Violación de los DDHH en dictadura, ya que aún quedan secuelas (S2. P99).

La violación de los derechos ya que hubo mucha injusticia (S2. P133).

Esta sensación de impunidad e injusticia, se comprende dado que los estudiantes durante la $\mathrm{EDH}$ recibida, toman conocimiento y debaten sobre varios temas contingentes en torno a los DDHH, entre ellos: la existencia de casos en los que aún no se sabe la verdad de lo sucedido con las víctimas, la ausencia de justicia sobre los responsables de estos hechos, el silencio institucional de las fuerzas armadas y civiles, que impide esclarecer la verdad. La falta de arrepentimiento y de cooperación de los condenados para saber la verdad, el negacionismo en autoridades públicas, entre otros.

A continuación, se presentarán los resultados más relevantes y pertinentes para este estudio, de dos categorías emergentes.

A través de la EDH, la exigibilidad de los $\mathbf{D D H H}$, es una preocupación importante para los estudiantes, ya que emerge como una especie de garantía para el empoderamiento y para materializar el ejercicio de los derechos, cuando estos corren el riesgo de ser vulnerados. Tanto para los propios estudiantes, como sujetos de derechos,

Estoy más informada para exigir mis derechos y tomar mayor conciencia para respetar el de los demás (S1. P38).

Hacer valer nuestros derechos, y asi no permitir que se nos pase a llevar (S2. P157).

Así como para los futuros sujetos, grupos y comunidades de intervención, como lo señalan los siguientes relatos:

Orientar e informar a las personas sobre lo que le pueden exigir al estado y orientar en caso de vulneración de derechos (S1. P36).

Ayudar y concientizar a las personas de sus derechos y como éstos, pueden exigir su respeto (S3. P215).

Reeducar para que ellos puedan ejercer y conocer sus derechos (S2. P81).

[...] además promoviendo continuamente el ejercicio de los DDHH en todas las esferas, que las personas defiendan sus derechos (S2. P93).

Proteger los DDHH de las personas especialmente de los más vulnerados, todos deben ser conscientes de nuestros derechos (S3. P215).

Los resultados recién presentados, como señalan Galheigo (2007) y Algado \& Galheigo (2012), fortalecen la identidad en los estudiantes del rol de articuladores sociales, en la práctica profesional futura. Específicamente, fortaleciendo el acceso y ejercicio de los derechos a los sujetos en situación de vulneración y riesgo social. 
Por último, la categoría ciudadanía, se entiende para este estudio como un aspecto conformador de subjetividad para los estudiantes, dado que implica un desarrollo y construcción de sujetos de derechos conscientes de sí mismo y de los demás, así como críticos y reflexivos del contexto que habitan, cuyo anhelo es construir una sociedad basada en los principios y la práctica de los DDHH:

Saber que es importante vivir en base a esto [los $\mathrm{DDHH}]$, porque sin esto creo que perdemos la esencia (S1. P3).

Ahora veo a las personas como sujetos de derecho, incluyéndome, ya que antes no lo conocía, por lo tanto, no podía defenderlos (S1. P18).

Esto nos hace ser mejores personas, $y$ asi de a poco ir formando una mejor sociedad (S1. P57).

Me ha permitido reflexionar acerca de hechos de vulneración, educar y concientizar a los demás respecto a esto (S2. P177).

Ampliar mi marco de conciencia (S3. P271).

Según estos relatos la $\mathrm{EDH}$ en TO, permitiría construir en los estudiantes la conciencia ocupacional ${ }^{4}$ (Ramugondo, 2015). Ya que luego de la formación recibida, los $\mathrm{DDHH}$ son considerados un soporte para culminar con condiciones de vulneración y opresión, las que ocurren cotidiana y sistemáticamente, en grupos sociales desfavorecidos e incluso en ellos mismos.

Al mismo tiempo, los estudiantes reconocen que en el ejercicio profesional futuro, la $\mathrm{EDH}$ recibida les ha permitido considerar que la intervención de $\mathrm{TO}$ es un acto político, que requiere concientizar y concientizarse en la acción interventiva, como lo señalan los siguientes relatos:

Lo fundamental es entregar un enfoque politico a la ocupación, y a la relación que tendremos con nuestros usuarios (S1. P25).

Tendremos vinculos con personas que son vulnerados en sus derechos, y nosotros tendremos un rol politico (S2. P132).

[...] si todos tuvieran conciencia de sus derechos, [...] no ocurririan tantas injusticias y abusos por parte de personas e instituciones (S3. P212).

En este sentido, la EDH en TO promueve una de las ideas fuerzas de Galheigo (2011), quien señala que la apropiación de los DDHH como fundamento en la TO, requiere el desarrollo de una conciencia política por parte de los y las terapeutas ocupacionales.

${ }^{4}$ Este concepto será presentado y profundizado en la discusión que se desarrolla más adelante. 


\section{Discusión}

A continuación, se profundizarán aspectos que se consideran fundamentales en la formación profesional de TO.

\subsection{EDH y conciencia ocupacional}

Algunos de los propósitos de la EDH es formar sujetos que sean capaces de reflexionar y comprender en qué condiciones sociales y materiales se producen o ejercen los DDHH (Córdova \& Galheigo, 2015). En tal sentido, los participantes de este estudio perciben que la democracia en Chile, se ha construido al costo de la vulneración de los DDHH.

Dicho lo anterior, la EDH promueve la construcción de la conciencia ocupacional en los estudiantes de TO. Como plantea Ramugondo (2015), la conciencia ocupacional nos permite ser críticos ante las condiciones que generan opresión, malestar e injusticias. La EDH permitiría despertar la conciencia ocupacional, ya que construye sujetos más críticos de la realidad social y motivados por modificarla.

Según lo planteado por Ramugondo \& Kronenberg (2015), la EDH promovería el desarrollo de un reconocimiento de ser social, un ser que supera la preocupación por sí mismo para avanzar a la responsabilidad ética de ser con otros y para otros. Esta condición es fundamental para la construcción y desarrollo de la conciencia ocupacional, así como de la ciudadanía.

En el campo formativo profesional, lo anterior implica considerar que los DDHH guíen la relación docente-estudiantes, la relación entre pares, la promoción de decisiones colegiadas y participativas, que los $\mathrm{DDHH}$ sean el referente en las prácticas de todas las actividades de la vida universitaria. Con el propósito de inscribir y construir la posibilidad de otras éticas, de otras formas de relaciones basadas en los principios valóricos de los $\mathrm{DDHH}$, como el respeto, la dignidad, la libertad colectiva, la cooperación, la solidaridad, entre otros.

\subsection{EDH y politización}

Los resultados de esta investigación, indicarían que uno de los efectos en la EDH en los estudiantes, es la paradoja que provoca el hecho que los $\mathrm{DDHH}$, no se pueden ejercer adecuadamente o que aún se siguen vulnerando o violando, a pesar de lo importante que son para la protección de la dignidad humana, y para una buena convivencia en la comunidad.

Para resolver esta paradoja, se expone abordar desde una perspectiva política la $\mathrm{EDH}$, es decir, organizar experiencias de aprendizaje que permitan a los estudiantes, comprender y analizar las correlaciones de fuerzas presentes entre la lucha y resistencia de grupos, movimientos y colectividades por conquistar y ejercer sus derechos; los mecanismos que despliega el neoliberalismo para restringir o vulnerar el ejercicio de los derechos; y el rol del Estado en la protección y promoción de éstos.

En este marco, considerando lo que plantean autores como Córdova \& Algado (2010) y Algado \& Galheigo (2012), es necesario que los estudiantes comprendan la responsabilidad ética de posicionarse en la práctica profesional, junto a los que luchan 
o requieren luchar para ejercer sus derechos, con el anhelo de alcanzar la justicia, el bienestar social y un buen vivir.

Esta tarea, es un imperativo ético, en tanto el modelo neoliberal imperante en Chile, especialmente en el campo educativo, construye estudiantes universitarios individualistas, competitivos, sin memoria histórica y apolíticos (Rivas \& Acevedo, 2017).

Se propone para avanzar en esta dirección, a partir de lo que plantean Albán \& Rosero (2016), promover en TO una pedagogía del reaprendizaje, es decir, cuestionar los cimientos deshumanizantes del modelo capitalista neoliberal y su negación constitutiva de los DDHH, para desde ahí, considerar los contextos sociales como escenarios de aprendizaje, para la construcción de una sociedad que dignifique la vida de los sujetos y comunidades, así como la configuración de subjetividades que gocen plenamente sus derechos.

Para ello, es necesario considerar 2 aspectos fundamentales; primero, comprender que la EDH implicaría una responsabilidad ética de los académicos, por formar sujetos políticos en la formación universitaria de las y los futuros Terapeutas Ocupacionales (Valenzuela, 2017); y segundo, sería necesario para lograr este propósito, que en el ejercicio pedagógico se proporcionen espacios de dialogo, reflexión y problematización con los estudiantes de la realidad social que los constituye (Palacios \& Diaz, 2016).

Es decir, que la misma práctica pedagógica permita la construcción de una comunidad política, en la cual se estimule la comunicación de los anhelos, se aúnen voluntades y se habiliten espacios de decisión deliberativa y pluralista, al interior de las aulas, así como en los espacios formativos de encuentro con los sujetos, comunidades y colectivos de intervención.

\subsection{Feminismo y TO}

En la dimensión igualdad y no discriminación, son interesantes los resultados obtenidos a través de las encuestas, en torno a la categoría mujeres, ya que dan cuenta que la formación recibida en torno a la protección de los DDHH de la mujer, son necesarios pero insuficientes para superar los arquetipos hacia el género femenino en los estudiantes de TO.

En ese marco, se propone entonces incorporar los feminismos en la formación profesional, no solo por su historia como movimiento que lucha por la reivindicación de los DDHH de las mujeres. Si no que considerar los feminismos, como un fundamento para transformar las estructuras y prácticas patriarcales y capitalistas, que perpetúan los estereotipos e injusticias hacia la mujer, y otros grupos sociales como los pobres e indígenas (Richard, 2018; Rojas, 2018).

Complementando lo anterior, se propone explorar en la formación disciplinar los feminismos, ya que como señala Morrison $(2011,2016)$, permitiría superar la androgenización de la historia de la disciplina, incorporando el reconocimiento de las mujeres en el desarrollo disciplinar, para despatriarquizar los fundamentos de la TO.

Desde la opinión de Morrison \& Araya (2018), los feminismos en la formación de TO forjarían ampliar el horizonte del ejercicio profesional y mejorar la calidad de las acciones interventivas. Más aún, cuando en la actualidad la TO es convocada a intervenir en problemas sociales más complejos, que articulan distintos tipos de 
opresiones a las cuales están sometidas las mujeres, por ejemplo: raza, clase social y mujer; discapacidad, clase social y mujer; discapacidad, pueblos originarios y mujer; entre otras.

Estas expresiones de opresión implicarían abordar en la formación disciplinar su relación con los $\mathrm{DDHH}$, y como éstos podrían ser un soporte para superar estas opresiones hacia la mujer, es decir, el fundamento para otras éticas posibles construidas desde la negociación colectiva del que sufre, del que transporta el peso histórico de las desigualdades, del que por derecho requiere ser reivindicado, a partir de su propio ser, desde su propia identidad.

¿Cuáles serían algunas prácticas pedagógicas en los procesos de enseñanza y aprendizaje?

Se requeriría instalar una educación no sexista, donde el género y los feminismos sean un tema transversal en el currículo y los programas de estudios, así como, en las metodologías de enseñanza.

Lo anterior implica un uso respetuoso del lenguaje por parte de los académicos, evitar sarcasmos y chistes de tono sexual entre académicos y estudiantes, evitar resaltar un supuesto predominio intelectual de los hombres por sobre las mujeres, destacando las ideas entregadas por ambos de una manera equitativa. Trabajar el prejuicio de que TO es una carrera de mujeres, porque reproduce simbólica y materialmente el arquetipo de mujer cuidadora, entre otros aspectos del cotidiano universitario.

\section{Conclusion}

La $\mathrm{EDH}$ en $\mathrm{TO}$, se reconoce como un camino que obliga a continuar problematizando la necesaria relación de la TO con los DDHH, especialmente en Latinoamérica y en aquellos países con un fuerte desarrollo capitalista neoliberal.

La EDH permite a los estudiantes de TO, comprender la realidad social desde una perspectiva más amplia y compleja. Desarrollar una mirada crítica de sí mismos y de la realidad social; al mismo tiempo, les proporciona comprender su práctica profesional futura, como una posibilidad de crear una comunidad y sociedad más justa, digna y solidaria.

La EDH en TO, es un recurso potente no solo por el conocimiento que ellos proporcionan, sino también para las prácticas interventoras, para el respeto de la diversidad y para la transformación de las realidades socioculturales inmersas en la discriminación, la opresión y la vulneración social.

Los desafíos no están solo puestos en el aprendizaje cognitivo de los DDHH, sino que sobretodo en la incorporación de metodologías de aprendizaje, que permitan crear estrategias y prácticas que materialicen el ejercicio de los $\mathrm{DDHH}$ en los sujetos, comunidades y colectivos con los cuales la TO trabaja.

Esto significa entonces, pensar la formación de $\mathrm{DDHH}$ no como un contenido técnico, sino como un ejercicio de ciudadanía en el plan de estudio en su conjunto, es decir, en la docencia en aulas, en la gestión de proyectos sociales, en las prácticas profesionales, en la investigación, en las actividades extracurriculares, etc.

En cuanto a las propuestas emergentes de este estudio, una de ellas es considerar las epistemologías del Sur en la formación profesional, ya que éstas permitirían el análisis de las contradicciones de los procesos colonizantes/capitalistas/patriarcales de nuestros 
territorios, y las alternativas descolonizantes/anticapitalistas/feministas y su relación con los DDHH desde la interculturalidad, es decir, desde y con los pueblos originarios, mujeres, migrantes, juventudes, discapacidades, vejez, entre otros grupos vulnerados.

Otro tema que se formula considerar en la formación disciplinar, es la soberanía comunitaria. Esta propuesta surge, dado que en el contexto actual los espacios públicos se encuentran en un creciente proceso de privatización, que impide o limita la posibilidad de construir comunidades y ciudadanía.

Para lo anterior, se expone desarrollar metodologías como el aprendizaje de servicios, para que los estudiantes junto con las comunidades, mejoren y mantengan plazas, colaboren en la coordinación y organización de juntas de vecinos u otras organizaciones sociales, en la construcción de redes de organización y de redes sociales de apoyo, y así colaborar en la defensa del patrimonio cultural material e inmaterial local, así como de la memoria individual y colectiva, de los individuos y grupos a los que se acompaña. Lo anterior con el propósito de fortalecer los lazos y vínculos sociales, de las personas y colectivos en su vida cotidiana.

\section{Agradecimientos}

A todo el equipo de académicos y estudiantes de la escuela de Terapia Ocupacional de la Universidad Andrés Bello, que participaron en la innovación curricular. Especialmente al terapeuta ocupacional Claudio Inostroza, secretario académico de la carrera de Terapia Ocupacional en Concepción, por su dedicación en dicho proceso y por el apoyo entregado en esta investigación.

\section{Referencias}

Alarcón, A. H., Reyes Martínez, C., Roa Riffo, M., \& Sepúlveda Hernandez, S. (2017). Uso terapéutico del cannabis: una lucha desde la ocupación colectiva. Revista de Estudiantes de Terapia Ocupacional, 4(1), 53-72.

Albán, A., \& Rosero, J. (2016). Colonialidad de la naturaleza: ¿imposición tecnológica y usurpación epistémica? Interculturalidad, desarrollo y re-existencia. Nómadas, (45), 27-41.

Algado, S. S., \& Galheigo, S. (2012). Maestras de la terapia ocupacional. Sandra Galheigo: la poderosa emergencia de la terapia ocupacional social. Revista TOG, 9(15), 18-41.

Amezcua, M., \& Galvéz, A. (2002). Los modos de análisis en investigación cualitativa en salud: perspectiva crítica y reflexiones en voz alta. Revista Espanola de Salud Publica, 76(5), 423-436.

Azúa, E. (2011). La educación en derechos humanos. In Actas Seminario Internacional, Derechos Humanos y Universidad (pp. 31-36). Santiago: Instituto Nacional de Derechos Humanos.

Bianchi, P., \& Malfitano, A. (2017). Formación en terapia ocupacional en América Latina: ‘avanzamos hacia la cuestión social? World Federation of Occupational Therapists Bulletin, 73(1), 15-23. http://dx.doi.org/10.1080/14473828.2017.1293206.

Cerón, M. C. (2006). Metodologías de investigación social. Santiago: LOM Ediciones.

Chile. (2018a). Informe de matricula 2018. Recuperado el 22 de agosto de 2018, de http://www.mifuturo.cl/index.php/futuro-laboral/buscador-por-carrera-d-institucion

Chile. (2018b). Primer plan nacional de derechos humanos, 2018-2021. Chile: Ministerio de Justicia y Derechos Humanos. 
Alcances y desafíos de la Educación en Derechos Humanos en la formacion de terapeutas ocupacionales, a partir de la percepcion de los estudiantes

Comisión Nacional de Acreditación - CNA. (2018). Resolución exenta de acreditación institucional: acoge recurso de reposición presentado por la Universidad Andrés Bello. Santiago.

Córdova, A. G., \& Algado, S. S. (2010). Una terapia ocupacional basada en derechos humanos. Revista $T O G, 7(12), 1-25$.

Córdova, A. G., \& Galheigo, S. (2015). Reflexiones crítica sobre los derechos humanos: contribuciones de la terapia ocupacional latinoamericana. Boletín de la Federación Mundial de Terapeutas Ocupacionales, 71(2), 73-81. http://dx.doi.org/10.1179/1447382815Z.00000000023.

Crawford, E., Aplin, T., \& Rodger, S. (2017). Human rights in occupational therapy education: A step towards a more occupationally just global society. Australian Occupational Therapy Journal, 64(2), 129-136. http://dx.doi.org/10.1111/1440-1630.12321.

Dehays, M., Hichins, M., \& Vidal, V. (2013). Análisis del significado de las ocupaciones atribuidas a ser mujer y madre para un grupo de mujeres con discapacidad intelectual en la ciudad de Punta Arenas. Santiago: Instituto Nacional de Derechos Humanos.

Espinoza, I. M., \& La Pietra, V. R. (2017). Incorporación del enfoque de derechos humanos a través de la metodología de aprendizaje servicio, análisis desde la percepción de estudiantes de terapia ocupacional de la Universidad Central de Chile. Revista Latinoamericana en Discapacidad, Sociedady Derechos Humanos, 1(1), 63-75.

Estay, J. M. (2010). Los derechos sociales de prestación en la jurisprudencia chilena. Estudios Constitucionales, 8(2), 125-166.

Fernández, F. L. (2012). Conceptos de ciudadano, ciudadanía y civismo. Polis, 11(32), 269-304. http://dx.doi.org/10.4067/S0718-65682012000200014.

Fernández, M. (2016). Educación en derechos humanos en Argentina. Notas sobre el proceso de incorporación de los derechos humanos en los contextos educativos. Revista Latinoamericana de Derechos Humanos, 27(1), 143-166. http://dx.doi.org/10.15359/rldh.27-1.7.

Galheigo, S. (2007). Terapia ocupacional en el ámbito social: aclarando conceptos e ideas. In F. Kronenberg, S. Simó \& N. Pollard (Eds.), Terapia ocupacional sin fronteras: aprendiendo del espiritu de supervivientes (pp. 85-97). Buenos Aires: Médica Panamericana.

Galheigo, S. (2011). What needs to be done? Occupational therapy responsibilities and challenges regarding human rights. Australian Occupational Therapy Journal, 58(2), 60-66. http://dx.doi.org/10.1111/j.1440-1630.2011.00922.x.

Galindo, N., Castillo, C., Negrón, G., Obando, J., Palma, F., \& Yupanqui, A. (2016). Mujeres privadas de libertad y apego. Una intervención de terapia ocupacional en Magallanes. Revista Chilena de Terapia Ocupacional, 16(1), 147-157. http://dx.doi.org/10.5354/0719-5346.2016.41952.

García, O. L., \& García-Celay, I. M. (2015). Métodos de investigación en psicología y educación: las tradiciones cuantitativas y cualitativas. España: McGraw Hill Ediciones.

Gurdián-Fernández, A. (2007). El paradigma cualitativo en la investigación socio-educativa. San Jose: Colección.

Hocking, C., \& Townsend, E. (2015). Driving social change: occupational therapists' contributions to occupational justice. World Federation of Occupational Therapists Bulletin, 71(2), 68-71.

Instituto Nacional de Derechos Humanos - INDH. (2011). Primera encuesta nacional de derechos humanos en Chile. Santiago.

Instituto Nacional de Derechos Humanos - INDH. (2012). Informe anual 2012: situación de los derechos humanos en Chile. Santiago.

Instituto Nacional de Derechos Humanos - INDH. (2014). La educación en derechos humanos en universidades: Revisión crítica de cursos para la elaboración de un programa de formación básica. Santiago.

Jung, B., Salvatori, P., Tremblay, M., Baptiste, S., \& Sinclair, K. (2008). Inclusive occupational therapy education: an international perspective. World Federation of Occupational Therapists Bulletin, 57(1), 33-42. http://dx.doi.org/10.1179/otb.2008.57.1.007. 
Alcances y desafíos de la Educación en Derechos Humanos en la formacion de terapeutas ocupacionales, a partir de la percepcion de los estudiantes

Larraín, J. (2011). ¿América latina Moderna? Globalización e identidad. Santiago: LOM Ediciones.

Lopes, R. E., Malfitano, A. P. S., Silva, C. R., Borba, P. L. O., \& Hahn, M. S. (2012). Occupational therapy professional education and research in the social field. World Federation of Occupational Therapists Bulletin, 66(1), 52-57. http://dx.doi.org/10.1179/otb.2012.66.1.021.

Magendzo, A. (1998). La educación en derechos humanos. Reflexiones y retos para enfrentar un nuevo siglo. In United Nations Educational, Scientific and Cultural Organization - UNESCO. Proyecto principal de educación en América Latina y el Caribe (pp. 27-33). Santiago: Boletin.

Magendzo, A. (2003). Pedagogía crítica y educación en derechos humanos. Revista de Pedagogía Crítica Paulo Freire, 2(2), 19-27.

Magendzo, A. (2008). Pensamiento e ideas fuerza en la educación en derechos humanos en Iberoamérica. In United Nations Educational, Scientific and Cultural Organization - UNESCO. Ideas-fuerzas y pensamiento en la educación en derechos humanos en Iberoamérica (pp. 4-26). Santiago: Editorial SM.

Magendzo, A., \& Toledo, M. (2015). Educación en derechos humanos: estrategia pedagógica-didáctica centrada en la controversia. Revista Electrónica Educare, 19(3), 1-16. http://dx.doi.org/10.15359/ree.19-3.2.

Morrison, R. (2011). (Re)conociendo a las fundadoras y “madres” de la terapia ocupacional. Una aproximación desde los estudian feministas de la ciencia. Revista TOG, 8(4), 1-21.

Morrison, R. (2016). Los comienzos de la terapia ocupacional en Estados Unidos: una perspectiva feminista desde los estudios de Ciencia, Tecnología y Género (siglos XIX y XX). Historia Crítica, (62), 97-117.

Morrison, R., \& Araya, L. (2018). Feminismo(s) y terapia ocupacional. preguntas y reflexiones. Revista Argentina de Terapia Ocupacional, 4(2), 60-72.

Mousavi, T., Forwell, S., Dharamsi, S., \& Dean, E. (2015). The historical shift towards human rights in occupational therapy with special reference to the capabilities approach and its implications. World Federation of Occupational Therapists Bulletin, 71(2), 81-87.

Murcia, L. P., Yepes, R. U., \& Garavito, C. R. (2007). Los derechos sociales en serio: hacia un diálogo entre derechos y politicas públicas. Bogotá: Ediciones Antropos.

Nash, C. (2011). Diagnóstico sobre la incorporación de los derechos humanos en las carreras de derecho en Chile. In Instituto Nacional de Derechos Humanos - INDH (Ed.), Actas Seminario Internacional (pp. 47-60). Santiago de Chile: Universidad y Derechos Humanos, Instituto Nacional de Derechos Humanos.

Navarrete Salas, E., Cantero Garlito, P. A., Guajardo Córdoba, A., Sepúlveda Prado, R., \& Moruno Miralles, P. (2015). Terapia ocupacional y exclusión social: hacia una praxis basada en los derechos humanos. España: Editorial Segismundo.

Organización de Naciones Unidas - ONU. (1993). Declaración y Programa de Acción de Viena, 20 años trabajando por tus derechos. Colombia.

Organización de Naciones Unidas - ONU. (2005). Documento final de la Cumbre Mundial 2005. Ginebra.

Organización de Naciones Unidas - ONU. (2010). Proyecto de plan de acción para la segunda etapa (20102014) del Programa Mundial para la educación en derechos humanos. Ginebra: Consejo de Derechos Humanos.

Organización de Naciones Unidas - ONU. (2011). Declaración de las Naciones Unidas sobre educación y formación en derechos humanos. Ginebra: Asamblea General de las Naciones Unidas.

Organización de Naciones Unidas - ONU. (2013). Proyecto de plan de acción para la tercera etapa (20152019) del Programa Mundial para la educación en derechos humanos. Ginebra: Consejo de Derechos Humanos.

Organización Mundial de Salud - OMS. (2015). Instrumento de calidad y derechos de la OMS: evaluando y mejorando la calidad y los derechos humanos en los establecimientos de salud mental y de apoyo social. Chile: Escuela de Salud Pública Dr. Salvador Allende, Universidad de Chile. 
Alcances y desafíos de la Educación en Derechos Humanos en la formacion de terapeutas ocupacionales, a partir de la percepcion de los estudiantes

Ortiz, J., Leiva, N., \& Robles, V. (2014). El Impacto que genera el diagnóstico de VIH en mujeres trabajadoras (Tesis de pregrado). Universidad Central de Chile, Santiago.

Ospina Rave, E. O., Sandoval, J. J., Aristizabal Botero, C. A., \& Ramirez Gomez, M. C. (2005). La escala de Likert en la valoración de los conocimientos y las actitudes de los profesionales de enfermería en el cuidado de la salud. Investigacion y Educacion en Enfermeria, 23(1), 14-29.

Oyarzún, E., Acevedo, C., Olivares, D., Palacios, M., \& Méndez, P. (2010). Sistematización del día de la educación. In Anales del $15^{\circ}$ Congresso Mundial da Federación Mundial de Terapeutas Ocupacionales. Santiago: WFOT.

Palacios, M., \& Diaz, M. (2016). Challenges in the educations of occupational therapist, discussed from a critical perspective. In D. Sakellariu \& N. Pollard (Eds.), Occupational therapies without borders: integrating justice with practice (pp. 587-595). China: Elsevier.

Piškur, B. (2013). Social participation: redesign of education, research, and practice in occupational therapy. Scandinavian Journal of Occupational Therapy, 20(1), 2-8. http://dx.doi.org/10.3109/11038128.2012.744093.

Ramugondo, E. (2015). Occupational consciousness. Journal of Occupational Science, 22(4), 488-501. http://dx.doi.org/10.1080/14427591.2015.1042516.

Ramugondo, E., \& Kronenberg, F. (2015). Explaining collective occupations from a human relations perspective: bridging the individual-collective dichotomy. Journal of Occupational Science, 22(1), 316. http://dx.doi.org/10.1080/14427591.2013.781920.

Restrepo, M. (2009). Apuntes para una reflexión sobre pedagogía de los derechos humanos en Colombia. In United Nations Educational, Scientific and Cultural Organization - UNESCO. Ideas-fuerzas y pensamiento en la educación en derechos humanos en Iberoamérica (pp. 104-133). Santiago.

Richard, N. (2018). La insurgencia feminista de mayo. In F. Zeran (Ed.), La rebelión contra el patriarcado: mayo feminista (pp. 115-126). Santiago: Ediciones LOM.

Rivas, D. F., \& Acevedo, M. L. (2017). Reflexiones sobre el quehacer del estudiante de terapia ocupacional en una sociedad neoliberal. Revista de Estudiantes de Terapia Ocupacional, 4(1), 1-13.

Rivero, D. B. (2008). Metodología de la investigación. Mexico: Ediciones Shalom.

Rojas, C. (2018). La rebelión feminista: poco de novedad y mucho de herencia. In F. Zeran (Ed.), La rebelión contra el patriarcado: mayo feminista (pp. 127-136). Santiago: Ediciones LOM.

Sacavino, S. (2015). Pedagogía de la memoria y educación para el “nunca más” para la construcción de la democracia. Folios, (41), 69-85.

Sacavino, S., \& Candau, V. (2008). Educación en derechos humanos en Brasil, ideas fuerzas y perspectivas de futuro. In United Nations Educational, Scientific and Cultural Organization UNESCO. Ideas-fuerzas y pensamiento en la educación en derechos humanos en Iberoamérica (pp. 6882). Santiago: Editorial SM.

Sarmiento, B. M., Jara, R. M., Cáceres, D. P., \& Reyes, B. R. (2018). Percepción de la construcción de género en estudiantes de terapia ocupacional: una aproximación al género en la vida cotidiana. Cadernos Brasileros de Terapia Ocupacional, 26(1), 163-175. http://dx.doi.org/10.4322/25268910.ctoAO1124.

Silva, C. (2014). La educación en derechos humanos en universidades: revisión crítica de cursos para la elaboración de un programa de formación básica. Chile: Instituto Nacional de derechos Humanos.

Sinembargo TV. (2016). Seis tragedias que han hecho de México un "campeón" mundial en violaciones a $D D H H$. Recuperado el 20 de septiembre de 2018, de https://www.youtube.com/watch?v=xM3nwcL2RZM

Tavares, C. (2016). La enseñanza de derechos humanos en las escuelas: una mirada sobre la política educacional en el Estado de Pernambuco, Brasil. Revista Latinoamericana de Derechos Humanos, 27(1), 167-183.

Universidad Andrés Bello - UNAB. (2017). Aprueba nuevo plan de estudios de la carrera de terapia ocupacional, DUNo 2497/2017. Santiago. 
Universidad de las Américas. (2018). Perfil de egreso completo. Recuperado el 3 de julio de 2018, de https://www.udla.cl/portales/tp9e00af339c16/perfilegreso/terapia-ocupacionalUDLA_PE_201810_204.pdf

Universidad San Sebastián. (2018). Perfil del egresado. Recuperado el 3 de julio de 2018, de http://www.uss.cl/ciencias-salud/carrera/terapia-ocupacional/descripcion/

Valderrama, C., Pino, J., Guzman, S., Zolezzi, R., Vera, R., Seguel, P., \& Palma, D. (2015). Articulando la academia con la intervención comunitaria en salud mental: experiencia desde una terapia ocupacional social. Revista Chilena de Terapia Ocupacional, 15(2), 1-17.

Valenzuela, D. G. (2017). Terapia ocupacional, discapacidad y género: la interseccionalidad como apertura hacia reflexiones pendientes. Revista Ocupación Humana, 17(2), 34-45.

Vega, C. C., \& Nogueira, J. L. L. (2018). Pedagogía de la memoria y escritura performativa en la educación en derechos humanos. Revista Latinoamericana de Derechos Humanos, 29(1), 35-50. http://dx.doi.org/10.15359/rldh.29-1.2.

Whiteford, G. (2017). Participation in higher education as social inclusion: an occupational perspective. Journal of Occupational Science, 24(1), 54-63. http://dx.doi.org/10.1080/14427591.2017.1284151.

Yupanqui, A., González B, M. Á., Llancalahuén V, M., Quilodrán O, W., \& Toledo A, C. (2016). Lenguaje, discriminación y discapacidad en el contexto educativo de Magallanes: un enfoque de derechos humanos desde la terapia ocupacional. Magallania, 44(1), 149-166. http://dx.doi.org/10.4067/S0718-22442016000100009.

\section{Autor para la correspondencia}

Cristian Mauricio Valderrama

e-mail: cvalderrama@unab.cl 